\title{
777.
}

\section{A SOLVABLE CASE OF THE QUINTIC EQUATION.}

[From the Quarterly Journal of Pure and Applied Mathematics, vol. xvirI. (1882), pp. 154-157.]

THE roots of the general quintic equation

may be taken to be

$$
\begin{aligned}
& (a, b, c, d, e, f \gamma x, 1)^{5}=0 \\
& -\frac{b}{a}+B+C+D+E \\
& -,+\omega^{4},+\omega^{3},+\omega^{2},+\omega, \\
& -,+\omega^{3},+\omega,+\omega^{4},+\omega^{2}, \\
& -"+\omega^{2},+\omega^{4},+\omega, "+\omega^{3}, \\
& -,+\omega,+\omega^{2},+\omega^{3},+\omega^{4},
\end{aligned}
$$

where $\omega$ is an imaginary fifth root of unity; and if one of the four functions $B$, $C, D, E$ is $=0$, say if $E=0$ (this implies of course a single relation between the coefficients), then the equation is solvable.

Writing $x=\xi-\frac{b}{a}$, we have

where

$$
(a, b, c, d, e, f)\left(\xi-\frac{b}{a}, 1\right)^{5}=\left(a^{\prime}, 0, c^{\prime}, d^{\prime}, e^{\prime}, f^{\prime} \gamma \xi, 1\right)^{5}
$$

$$
\begin{aligned}
& a^{\prime}=a, \\
& a c^{\prime}=a c-b^{2}, \\
& a^{2} d^{\prime}=a^{2} d-3 a b c+2 b^{3}, \\
& a^{3} e^{\prime}=a^{3} e-4 a^{2} b d+6 a b^{2} c-3 b^{4}, \\
& a^{4} f^{\prime}=a^{4} f-5 a^{3} b e+10 a b^{2} d-10 a b^{2} c+4 b^{5},
\end{aligned}
$$

and the roots of the new equation

$$
\left(a^{\prime}, 0, c^{\prime}, d^{\prime}, e^{\prime}, f^{\prime} \gamma \xi, 1\right)^{5}=0
$$


have the above-mentioned values, omitting therefrom the terms $-\frac{b}{a}$; we find without difficulty

$$
\begin{aligned}
2 \frac{c^{\prime}}{a^{\prime}} & =-B E-C D, \\
2 \frac{d^{\prime}}{a^{\prime}} & =-B^{2} D-B C^{2}-C E^{2}-D^{2} E \\
\frac{e^{\prime}}{a^{\prime}} & =-B^{3} C-B^{2} E^{2}+B C D E+B D^{3}+C^{3} E+C^{2} D^{2}-D E^{2}, \\
\frac{f^{\prime}}{a^{\prime}}= & -B^{5}+5 B^{3} D E-5 B^{2} C^{2} E-5 B^{2} C D^{2}+5 B C^{3} D+5 B C E^{3} \\
& -5 B D^{2} E^{2}-C^{5}+5 C D^{3} E-5 C D^{2} E^{2}-D^{5}-E^{5},
\end{aligned}
$$

and hence, when $E=0$, we have

$$
\begin{aligned}
& 2 \frac{c^{\prime}}{a^{\prime}}=-C D \\
& 2 \frac{d^{\prime}}{a^{\prime}}=-B^{2} D-B C^{2}, \\
& \frac{e^{\prime}}{a^{\prime}}=-B^{3} C-B D^{3}-C^{2} D^{2}, \\
& \frac{f^{\prime}}{a^{\prime}}=-B^{5}-5 B^{2} C D^{2}+5 B C^{3} D-C^{5}-D^{5},
\end{aligned}
$$

or, as these may be written,

$$
\begin{array}{ll}
-2 \frac{c^{\prime}}{a^{\prime}} & =C D \\
-2 \frac{d^{\prime}}{a^{\prime}} & =B^{2} D+B C^{2}, \\
-\frac{e^{\prime}}{a^{\prime}}-4 \frac{c^{\prime 2}}{a^{\prime 2}} & =B^{3} C-B D^{3} \\
-\frac{f^{\prime}}{a^{\prime}} & =B^{5}+C^{5}+D^{5}-10 \frac{c^{\prime}}{a^{\prime}}\left(B^{2} D-B C^{2}\right),
\end{array}
$$

equations which imply a single relation between the coefficients $a^{\prime}, c^{\prime}, d^{\prime}, e^{\prime}, f^{\prime}$. Supposing this satisfied, we may attend only to the first three equations; or, writing for convenience,

$$
\begin{array}{ll}
\gamma=-2 \frac{c^{\prime}}{a^{\prime}}, & =-\frac{2}{a^{2}}\left(a c-b^{2}\right), \\
\delta=-2 \frac{d^{\prime}}{a^{\prime}}, & =-\frac{2}{a^{3}}\left(a^{2} d-3 a b c+2 b^{3}\right), \\
\theta=-\frac{e^{\prime}}{a^{\prime}}-4 \frac{c^{\prime 2}}{a^{\prime 2}}, & =-\frac{1}{a^{4}}\left\{a^{2}\left(a e-4 b d+3 c^{2}\right)+\left(a c-b^{2}\right)^{2}\right\}
\end{array}
$$

the equations are

$$
\begin{aligned}
& \gamma=C D, \\
& \delta=B\left(B D+C^{2}\right), \\
& \theta=B\left(D^{3}-B^{2} C\right) .
\end{aligned}
$$


The first equation gives $C=\frac{\gamma}{D}$, and substituting this value in the other two equations, we have

$$
\begin{aligned}
& B^{2} D^{3}+B \gamma^{4}-\delta D^{2}=0 \\
& B^{3} \gamma+B D^{4}+\theta D=0
\end{aligned}
$$

Eliminating $B$, the result is obtained in the form Det. $=0$. where in the last column of the determinant each term is divisible by $D$; and omitting this factor, the result is

$$
\left|\begin{array}{rrrr} 
& D^{3}, & \gamma^{2}, & -\delta D \\
D^{3}, & \gamma^{2}, & -\delta D^{2}, & \\
D^{3}, & \gamma^{2}, & -\delta D^{2}, & \\
\gamma, & 0, & -D^{4}, & \theta \\
\gamma, & 0, & -D^{4}, & \theta D,
\end{array}\right|=0 .
$$

If, in order to develope the determinant, we consider it as a sum of products, each first factor being a minor composed out of columns 1 and 2, and the second factor being the complementary minor composed out of columns $3,4,5$ (the several products being of course taken each with its proper sign), the expansion presents itself in the form

$$
\begin{aligned}
& D^{3} \gamma\left(-\theta \delta \gamma^{2} D^{2}+\delta^{2} D^{7}\right) \\
- & D^{6}\left(-\theta \gamma^{2} D^{4}+\delta D^{9}-\theta^{2} D^{4}\right) \\
- & \gamma D^{3} \cdot-\delta D^{2}\left(\delta D^{5}-\theta \gamma^{2}\right) \\
+ & \gamma^{3}\left(\gamma^{2} \delta D^{5}-\theta \delta D^{5}-\theta \gamma^{4}\right) \\
- & \gamma^{2} \delta^{3} D^{5} .
\end{aligned}
$$

Hence, collecting, and changing the sign of the whole expression, we obtain

$$
\delta D^{15}-\left(2 \gamma \delta^{2}+\gamma^{2} \theta+\theta^{2}\right) D^{10}+\left(-\gamma^{3} \delta+3 \gamma \delta \theta+\delta^{3}\right) \gamma^{2} D^{5}+\gamma^{7} \theta=0,
$$

a cubic equation for $D^{5}$. We have then as above $C=\frac{\gamma}{D}$, and $B$ is given rationally as the common root of the foregoing quadric and cubic equations satisfied by $B$.

Substituting for $\gamma, \delta, \theta$ their values in terms of the original coefficients, the equation for $D^{5}$ becomes

$$
\begin{array}{r}
2\left(a^{2} d-3 a b c+2 b^{3}\right)(a D)^{15} \\
+\left\{\begin{array}{c}
a^{4}\left(a e-4 b d+3 c^{2}\right)^{2} \\
+a^{2}\left(a c-b^{2}\right)^{2}\left(a e-4 b d+3 c^{2}\right) \\
-16\left(a c-b^{2}\right)\left(a^{2} d-3 a b c+2 b^{3}\right)^{2}
\end{array}\right\}(a D)^{10} \\
+4\left(a c-b^{2}\right)^{2}\left\{\begin{array}{cc}
28 & \left(a c-b^{2}\right)^{3}\left(a^{2} d-3 a b c+2 b^{3}\right) \\
+12 a^{2}\left(a c-b^{2}\right) & \left(a^{2} d-3 a b c+2 b^{3}\right)\left(a e-4 b d+3 c^{2}\right) \\
+8 & \left(a^{2} d-3 a b c+2 b^{3}\right)^{3}
\end{array}\right\}(a D)^{5} \\
-128\left(a c-b^{2}\right)^{7}\left\{a^{2}\left(a e-4 b d+3 c^{2}\right)+\left(a c^{2}-b^{2}\right)^{2}\right\}=0,
\end{array}
$$

and the solution of the given quintic equation thus ultimately depends upon that of this cubic equation. 